\title{
Acute Myeloid Leukemia in Patients Older than 60 Year
}

\author{
Nahla AM Hamed* \\ Department of Hematology, Alexandria University, Egypt
}

Submission: September 04, 2018; Published: October 09, 2018

*Corresponding author: Nahla AM Hamed, Faculty of Medicine, Department of Hematology, Alexandria University, Egypt, Email: drhamedn@hotmail.com

\section{Abstract}

Intensive remission induction chemotherapy should be considered by clinicians as the first choice for selected very old AML patients whenever feasible on clinical grounds. Early death in most studies does not seem to play a major role in the inferior outcome of elderly AML patients. The early death rate in intensively-treated patients has decreased considerably over the last 2 decades, most probably owing to better supportive care.

Abbreviations: AML: Acute Myeloid Leukemia; OS: Overall Survival; CR: Complete Remission; HMAs: Hypomethylating Agents; DAC: Decitabine; AZA: Azacitidine; LDAC: Low-Dose Cytarabine; BSC: Best Supportive Care; ICT: Intensive Chemotherapy; RIC: Reduced Intensity Conditioning

\section{Introduction}

The median age of AML at diagnosis is 67 years with $54 \%$ of patients diagnosed at 65 years or older (and approximately a third diagnosed at $>75$ years of age) [1]. Older AML, defined as patients aged $>60$ years, has been one of the most challenging subsets to stratify and derive treatment decisions [2].

\section{Determinants of treatment failure in older AML}

The determinants of treatment success and failure remain partly understood. A combination of patient-related and specific disease-related factors are more common among this aging population and correlate markedly with treatment failure (i.e., primary resistance and relapse after induction therapy). They include comorbid conditions, performance status, decreased drug clearance and prolonged exposure to chemotherapeutics resulting from pharmacokinetic and pharmacodynamic changes, lesstolerability to infectionsdueto decreasedimmune competence of elderly patients, psychosocial factors (cognitive decline, social isolation, and, often, lack of caretakers), multidrug resistant abilities of the leukemia cells to expel the chemotherapeutics, antecedent hematologic disorders [3] (previous MDS, chronic myelomonocytic leukemia, myeloproliferative neoplasm), prior exposure to cytotoxic therapy for other disorders [4] and higher frequencies of adverse cytogenetics and unfavorable molecular aberrations [3].

\section{Cytogenetic and molecular changes}

The percentage of favorable cytogenetics $(t(8 ; 21)$, in$\mathrm{v}(16) / \mathrm{t}(16 ; 16)$, and $\mathrm{t}(15 ; 17))$ decreased to a low percentage among the oldest age groups $(<5 \%$ for patients older than 70 years;). No significant difference in frequency between patients who were not in the favorable or adverse cytogenetic group in different age groups. The rate of unfavorable risk cytogenetics increased continuously up to $36 \%$ in patients older than 85 years [5].

Chromosome 5 and 7 aberrations increase in the oldest age groups while complex karyotypes increase continuously up to $28 \%$ in the oldest age group. Trisomy 8 as a sole aberration was found more frequent in older patients than other age groups [5]. Monosomal karyotype increased with age to $20 \%$ in patients older than 60 years [1]. The median age of patients with chromosomal aneuploidy and TP53 mutations is 58 years as opposed to 49 years for the patients with aneuploidy alone. Chromosomal aneuploidy, and TP53 mutations are enriched in older AML ( $49 \%$ of $>60$ year-olds belong to either group) [2].

The high frequency of complex karyotypes and unbalanced aberrations in older patients indicates multiple genetic events, including epigenetic changes during a lifetime, a prolonged duration of exposure to environmental carcinogens which correlates with the number of mutations (especially in patients older than 60 years), and an accumulation of mutations from genetic error events in cell division. It has been demonstrated that clonal hematopoietic cells from normal individuals also accumulate mutations as a function of age [5]

RUNX1, ASXL1 and TP53 mutations (ELN adverse-risk category) are common in very old patient [6]. The frequency 
of NPM1-positive cases increases up to $43 \%$ among 60 -yearold patients and decreases afterwards. The percentage of NPM1/ FLT3-positive patients is lower (up to 19\%), but the age distribution remains similar to that for all NPM1-positive patients [5].

\section{Treatment options}

Older AML patients can be offered one of the following treatment options: standard induction treatment consisting mostly of $3+7$ regimen of an anthracycline and Ara-C; hypomethylating agents; investigational drugs within a clinical trial; low-dose Ara-C (LDAC); Best Supportive Care (BSC) with oral cytostatic drugs like hydroxyurea and/or transfusions [3].

\section{Standard induction treatment}

The choice between intensive, potentially curative chemotherapy or a palliative therapeutic approach in old AML patients should be guided by an evidence-based, individualized assessment of the potential risks (e.g., treatment-related mortality) and benefits of intensive chemotherapy [6].

The recommendation of intensive chemotherapy largely depends on assessment of the clinical performance and medical condition as judged by the medical doctor. Risk-scoring systems could be instrumental in this regard. Geriatric assessments with a focus on cognitive and physical function by either objective measurements or self-reported measures have not been widely studied. However, early studies suggest that they have added value in comparison with performance status and comorbidity evaluations, and thus they may improve the prediction of survival in older patients receiving intensive chemotherapy [3].

\section{Which older age patient can receive intensive treat- ment and not experience prohibitive toxicity?}

Two models predict the probability of achieving a CR and the risk for early death of untreated medically fit elderly AML patients. The factors included in the first algorithm are body temperature $\left(\leq 38{ }^{\circ} \mathrm{C}\right.$ and $>38{ }^{\circ} \mathrm{C}$ ), hemoglobin levels ( $\leq 10.3$ and $>10.3 \mathrm{~g} / \mathrm{dL})$, platelet counts $(\leq 28 \mathrm{~K},>28 \mathrm{~K}-\leq 53 \mathrm{~K},>53 \mathrm{~K}-\leq 10 \mathrm{~K}$ and $>10 \mathrm{~K}$ counts $/ \mathrm{mcL}$ ), fibrinogen levels $(\leq 150$ and $>150 \mathrm{mg} /$ $d L)$, age at diagnosis (60-64, >64-67, >67-72 and $>72$ years) and the type of leukemia (de novo and secondary). The other model includes performance score, age, platelet count, serum albumin, presence or absence of secondary AML, WBC count, peripheral blood blast percentage and serum creatinine (AUC 0.8-0.83) [1].

Patient registries and randomized trials suggest that intensive induction therapy with 2 courses of cytarabine and daunorubicin [1] can prolong OS at least in a subset of elderly patients aged 70-79 years. A trial of induction chemotherapy in this age group showed that favorable- and intermediate risk cytogenetics, IDH1 wild type status and good performance status may achieve relatively favorable OS, long-term survival after induction chemotherapy compared to the adverse risk group. The candidates' patients might be those with absent medical contraindications [6]. Standard dose of cytarabine and anthracycline with midostaurine may be considered in patients with FLT3 positive mutation AML [1]. Because many older patients have some evidence of antecedent myelodysplasia, full normalization of peripheral blood counts often does not occur even if therapy clears the marrow blasts [1].

CR was achieved in $45 \%$ to $65 \%$ of older newly diagnosed AML adults treated with standard ICT [7]. No survival advantage was observed in patients aged 65 to 70 years old who received two intensive consolidation courses rather than one single intensive consolidation course in the ALFA 9801 Trial [7]. RIC allogeneic HCT is considered an additional option for postremission therapy in patients aged 60 years and older with minimal comorbidities and in CR to induction therapy [1]. Prolonged survival has been reported in older patients receiving AZA maintenance after achieving MRD negative remission after ICT [7].

\section{DNA methyltransferase 1 inhibitors (HMAs)}

HMAs is a backbone for combination strategies in older patients and in those with adverse genetics or comorbidity that make them at high risk for treatment failure and/or unacceptable toxicity with standard intensive approaches [8]. Both HMAs, Decitabine (DAC) and Azacitidine (AZA) can improve median and short-term OS compared with LDAC or Best Supportive Care (BSC) only, even if the cure rates has not improved [7].

\section{Low-dose Ara-C (LDAC)}

Favorable or NK-AML patients show CR rate of $18 \%$ with subcutaneous LDAC (20 mg twice daily for 10 consecutive daily every 4-6 weeks) and a survival benefit compared with CR rate of $1 \%$ with hydroxyurea at a dose that maintain $\mathrm{WBC}<10.000 / \mathrm{mcL}$ [1]. The combination of clofarabine and LDAC appears promising in older patients who may not be suitable for standard induction therapy [1].

A wait-and-see approach with supportive care and cytoreduction with hydroxyurea does not improve quality of life or prolong survival of AML patients because none of the basic medical problems are tackled [3].

\section{Novel agents}

- $\quad \mathrm{GO}$ is a conjugate of an anti- CD33 monoclonal antibody and the cytotoxic agent, calechiamicin. The cell surface antigen CD33 is expressed in $>90 \%$ of AML patients. The FDA has made it available on a "compassionate use" basis for patients with suboptimal responses, including persistence of MRD after initial therapy [9].

- $\quad$ SGN-CD123A is an antibody-drug conjugate with the antibody directed at CD123. CD123 is more restrictively expressed in the healthy hematopoietic compartment, which might decrease on-target off-leukemia toxicities. It has been tested in relapsed/refractory AML in phase I trial (NCT02848248) and in combination with AZA or DAC in newly diagnosed older AML patients (CASCADE study) in 
phase III study (NCT02785900). Hepatotoxicity, including veno-occlusive disease is a major concern, particularly when combined with allogeneic HSCT before or after the treatment.

- CPX-351 (an encapsulation in nanoscale liposomes of cytarabine and daunorubicin at a synergistic 5:1 molar Ratio) may improve therapy of older patients with high-risk features [4] and was also efficacious in older patients with secondary AML [1].

\section{Special situations}

- Treatment options of older adults with unfavorable cytogenetic/ molecular markers, antecedent hematologic disorder, or therapy-related AML include clinical trial, lowerintensity therapy with HMA, standard infusional cytarabine and anthracycline or clofarabine with or without standarddose cytarabine [1].

- $\quad$ Patients with adverse risk cytogenetics, IDH1 mutations or poor performance status did not benefit from intensive induction therapy and might fare better with alternative strategies such as HMAs, or novel targeted agents that have recently been approved or are currently under development [6].

- $\quad$ Allogeneic HSCT is considered to be probably the best option to prevent AML recurrence in selected patients with intermediate- or adverse-risk AML [7].

How to manage older AML patients with high WBC at diagnosis remains an open issue, because these patients appear to poorly respond to both chemotherapy and HMAs. Combination with kinase inhibitors represents an interesting approach because they frequently present activating kinase mutations, such as FLT3 internal tandem duplications [7].

\section{Relapsed AML}

The physically fit relapsed patients 60 years or older: may be offered the following options: 1) therapy on a clinical trial (strongly preferred); 2) chemotherapy followed by RIC allogeneic HCT (transplant should be considered if the patient has entered remission or in the context of clinical trial; 3) retreatment with the initial successful induction regimen for patients with long initial induction duration (relapse $>12$ months) [1].

The unfit relapsed elderly AML patients: are often selected for clinical trials with new therapeutic agents [3]. Many new drugs have failed approval, partly because these drugs may be tested in suboptimal setting. Relapsed/refractory AML in the older patient population contains some of the most notoriously resistant leukemias [3]. AZA and DAC induce CR rates of $16 \%$ to $21 \%$ and median survival times of 6 to 9 months in older patients with relapsed/refractory AML [4].

\section{Prognosis}

It remains unclear which genetic and clinical factors are relevant to identify those elderly patients most likely to benefit from, and least likely to be harmed by, induction chemotherapy
[6]. The median OS in patients aged $\geq 75$ years, regardless of therapy type, was 4.3 months with a 3 -year OS of $14 \%$, compared to a median OS of 10 months and a 3 -year OS of $20 \%$ in patients aged $<75$ years [6]. The adverse outcomes associated with older age are in part explained by the aggregation of poor cytogenetics and, importantly, gene mutations [2].

\section{Cytogenetics}

Good-risk cytogenetics (core-binding factor leukemias) has a distinctly favorable impact in older AML patients. Conversely, Adverse-risk cytogenetics (MRC classification) are associated with shorter OS [6]. Monosomal karyotypes carry adverse prognostic value [3]. The 2-year OS was significantly decreased among patients with monosomal karyotype compared with patients without this abnormality ( $7 \%$ vs $22 \%)$. Similar outcomes were observed within the subgroup of patients with complex karyotype [1].

Chromosomal aneuploidy and TP53 mutations maintain an independent adverse prognostic potential when age is accounted for [2].

\section{Molecular markers}

Favorable molecular markers established in younger patients (e.g., mutated NPM1 without or with low allelic ratio FLT3-ITD, biallelic CEBPA mutations) have weaker prognostic relevance in elderly patients [6]. NPM1 and FLT3-ITD mutations had no significant impact on OS [6]. The impact of FLT3-ITD mutation as a prognostic factor is reduced or absent in elderly patients [6].

Various studies have established the favorable effect of the NPM1-mutant genotype in the absence of FLT3 gene mutations for older AML patients treated with intensive chemotherapy protocols [3]. Alternately, in some studies, FLT3-ITD, DNMT3A, and ASXL1 gene mutations seem to confer a negative effect on response and survival in older patients with normal cytogenetics [3].

Some driver gene mutations, such as DNA-methyltransferase 3A (DNMT3A), TET2, SRSF2, and ASXL1, may be detected in seemingly healthy patients before overt AML development. Such preleukemic clones are more frequent in older patients. Their persistence during CR seems to represent a poor prognostic factor associated with higher incidence of relapse in these patients [7].

\section{Conclusion}

Gene-expression levels have to be evaluated in elderly AML patients for their prognostic value. In addition, well-designed clinical trials were recommended to include older AML patients whenever possible. New drugs in fit, elderly AML patients and in distinctly genomic-defined AML could be informative.

\section{References}

1. NCCN Clinical Practice Guidelines in Oncology (NCCN Guidelines) (2018) Acute myeloid leukemia. Version 2.2018- august 1, 2018. NCCN. org. 
2. Moarii M, Papaemmanuil E (2017) Classification and risk assessment in AML: integrating cytogenetics and molecular profiling. Hematology Am Soc Hematol Educ Program 2017(1): 37-44.

3. Ossenkoppele G, L"owenberg B (2015) How I treat the older patient with acute myeloid leukemia. Blood 125(5): 767-774.

4. D"ohner H, Estey E, Grimwade D, Amadori S, Appelbaum FR, et al (2017) Diagnosis and management of AML in adults: 2017 ELN recommendations from an international expert panel Blood 129(4) 424-447.

5. Creutzig U, Zimmermann M, Reinhardt D, Rasche M, von Neuhoff $C$ et al. (2016) Changes in cytogenetics and molecular genetics in acute myeloid leukemia from childhood to adult age groups. Cancer 122(24) 3821-3830.
6. Prassek VV, Rothenberg-Thurley M, Sauerland MC, Herold T, Janke H, et al. (2018) Genetics of acute myeloid leukemia in the elderly: mutation spectrum and clinical impact in intensively treated patients aged $\geq 75$ years. Haematologica 191536.

7. Dombret H, Itzykson R (2017) How and when to decide between epigenetic therapy and chemotherapy in patients with AML. Hematology Am Soc Hematol Educ Program 2017(1): 45-53.

8. Perl AE (2017) The role of targeted therapy in the management of patients with AML. Hematology Am Soc Hematol Educ Program 2017(1): 54-65.

9. Estey EH (2016) Acute myeloid leukemia: 2016 Update on riskstratification and management. Am J Hematol 91(8): 824-846.

\section{Your next submission with Juniper Publishers will reach you the below assets}

- Quality Editorial service

- Swift Peer Review

- Reprints availability

- E-prints Service

- Manuscript Podcast for convenient understanding

- Global attainment for your research

- Manuscript accessibility in different formats

( Pdf, E-pub, Full Text, Audio)

- Unceasing customer service

Track the below URL for one-step submission

https://juniperpublishers.com/online-submission.php 